$\$$ Research Square

\title{
Assessment of researches and case studies on Cloud Manufacturing: A bibliometric analysis
}

Daniel Alexandre Morelli ( $\nabla$ damorelli@gmail.com )

University of Campinas-UNICAMP

\section{Paulo Sergio de Arruda Ignacio}

University of Campinas-UNICAMP https://orcid.org/0000-0003-4688-8693

\section{Research Article}

Keywords: Cloud manufacturing, Industry 4.0, Bibliometric analysis, Case studies, Real-life applications

Posted Date: June 14th, 2021

DOI: https://doi.org/10.21203/rs.3.rs-620316/v1

License: (c) (i) This work is licensed under a Creative Commons Attribution 4.0 International License. Read Full License

Version of Record: A version of this preprint was published at The International Journal of Advanced Manufacturing Technology on August 4th, 2021. See the published version at https://doi.org/10.1007/s00170-021-07782-0. 


\title{
Assessment of researches and case studies on Cloud Manufacturing: A bibliometric analysis
}

\author{
Daniel Alexandre Morelli • Paulo Sergio de Arruda Ignacio
}

Received: date / Accepted: date

\begin{abstract}
Cloud computing technology has been studied in the context of industry 4.0 as a tool applied to manufacturing services and resources. Such concept is widely known as Cloud Manufacturing. This paper aims at mapping the current state of academic researches on this field, promoting the understanding of trends, references and practical applications in real-life conditions. A bibliometric analysis was conducted using two different databases - Scopus and Web of Sciences - and VOSviewer's text mining tools and techniques. From a sample of 1,420 papers, this study identified the countries which had the largest volume of publications, the main journals related to the subject, the most influent articles, and four clusters by keywords occurrences: (i) "Optimization of manufacturing processes", (ii) "Collaborative networks of manufacturing resources and services", (iii) "Industry 4.0 and cloud computing systems", and (iv) "Data reliability and cyber-security". Finally, this work selected and analyzed the 159 articles with applied case studies, in order to stratify and to understand the most common approaches within the four pre-established categories. This article can contribute to researchers and developers searching for successful practical applications in digitalization of manufacturing chains, as well as to those who are looking for gaps in the still unexplored fields of Cloud Manufacturing. Both the assessment and the categorization of the case studies about Cloud Manufacturing is a differential in this article.
\end{abstract}

Keywords Cloud manufacturing · Industry 4.0 - Bibliometric analysis · Case studies · Real-life applications

Industrial Engineering Reasearch Center, School of Applied Sciences, University of Campinas-UNICAMP, R. Pedro Zaccaria, 1300, Limeira-SP, Brazil

E-mail: damorelli@gmail.com·psai@unicamp.br

\section{Introduction}

The manufacturing industry, in the context of the fourth industrial revolution, must be capable of applying advanced technologies and knowledge, integrating the automation of its machines, equipment and sensors. Such integration should include not only the company's processes, systems and/or internal protocols, but also those of other companies that are part of its supply chain [1].

Academic researches and the industry's development departments have initiated to explore a set of innovative tools, among them, a manufacturing model based on the emerging cloud computing technology, integrating different sectors and companies on the network, through the virtualization and sharing of its resources and knowledge, and the communication between machines [2].

Built over web-based architectures, these models can generate value through collaborative processes between stakeholders, cost reduction and scalability [3]. Their proposition intends to promote performance improvement in a supply chain environment over the implementation of a digital and continuous information flow [4].

The application of these standards, which associate cloud computing advantages to manufacturing resources, started to be studied in 2010 and are called Cloud Manufacturing [5]. Since then, many researches have emerged in this field. Some of them are focused on theoretical frameworks, while others propose real-life practical applications through case studies.

A bibliometric analysis about the subject of Cloud Manufacturing was accomplished in this article, in order to assess academic researches and their case studies approaches, published until January 2021. 
This investigation aims at mapping the current state of a wide range of available researches in the field of Cloud Manufacturing, identifying publication trends, countries with the largest volume of papers, most relevant journals and influential articles, as well as the clusters of the keywords co-occurrences. This work then selected and analyzed the researches that included case studies, in order to categorize their approaches.

The contribution of this article is to generate relevant information to expand the knowledge about Cloud Manufacturing, contextualize trends of application tools, make successful practical solutions and relevant references available, as well as, identify gaps or improvement opportunities of studies in unexplored fields of Cloud Manufacturing.

\section{Literature Review}

Cloud computing technology represents a service model that enables users or enterprises to access a whole set of adaptable, configurable and available on-demand IT resources, in an agile way and with a low level of effort $[6]$.

According to Zárate and Mendoza [7], in a cloud computing architecture, all of the computing structures are treated as a service. Figure 1 shows the services delivered through cloud technology.

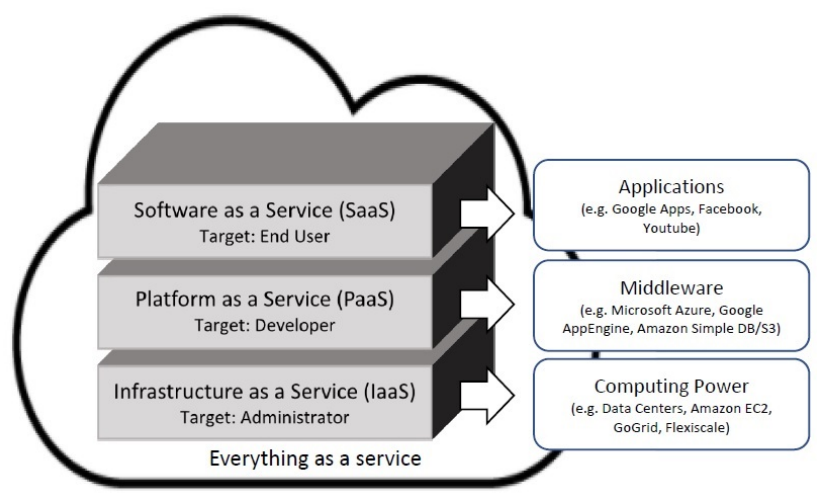

Fig. 1 Resources as a service in cloud computing [8]

- IaaS (Infrastructure as a Service), also known as hardware as a service (HaaS), delivers computing infrastructures, such as storage or data bases, using virtual machines (e.g., Amazon EC2, GoGrid, Flexiscale or Data Centers);

- PaaS (Platform as a Service) provides a system environment called middleware, driven to software development, as well as, testing and/or hosting of applications (e.g., Microsoft Azure, Google AppEngine or Amazon Simple DB/S3); and
- SaaS (Software as a Service) which offers a set of internet accessible applications to end users (e.g., Google Apps, Facebook, Youtube or Salesforce).

Cloud computing technologies applied specifically to the manufacturing sector is addressed by 3 main terms: Cloud Manufacturing, Cloud-based Manufacturing, or even Cloud-based Design and Manufacturing. By gathering the benefits of cloud computing, such as agility, flexibility, scalability and efficiency, many companies are being able not only to improve their own production processes, but also promote a greater integration and a more collaborative relationship with their business partners [9].

Based on networks, Cloud Manufacturing transforms manufacturing resources and capabilities into services performed through machine virtualization, which can be managed and operated on-demand by users for the whole life cycle of manufacturing [10].

As illustrated in figure 2, such process consist of three layers.

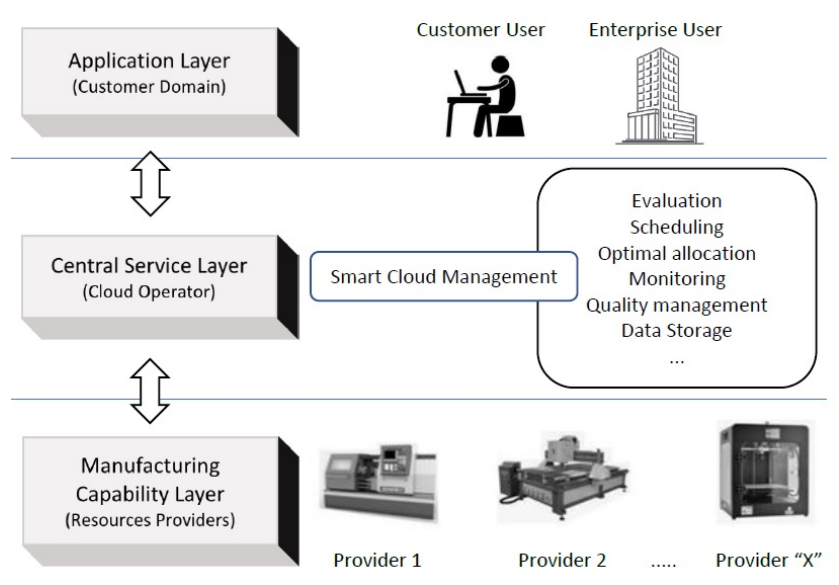

Fig. 2 The Cloud Manufacturing architecture model [11]

- Application Layer - This layer includes interfaces of users that need manufacturing services or resources to meet their customized demands. Those requests can be made by companies or individual users with access though the internet [12].

- Manufacturing Capability Layer - In this layer occurs the connection between the physical manufacturing machines and the cloud servers, by the virtualization of these resources [13]. Countless suppliers offer their specialized services and make their manufacturing machines available worldwide. Generally, these facilities are CNC machining centers, foundries industries, $3 \mathrm{D}$ printing, plastic injection molding, and other component production processes.

- Central Service Layer - In this layer happens the smart cloud solutions management, as matching be- 
tween customers' demands and physical resources available. This assessment enables production processes evaluation, scheduling, optimal resources allocation, monitoring, quality management, big data and real time information [11].

Figure 3 describes some of the potential applications enabled by the network connection involving different manufacturing companies. Cloud Manufacturing may be used to share resources and knowledge in order to be effective in the support of processes such as design, manufacturing, planning, controling, analysis and decision making. [14].

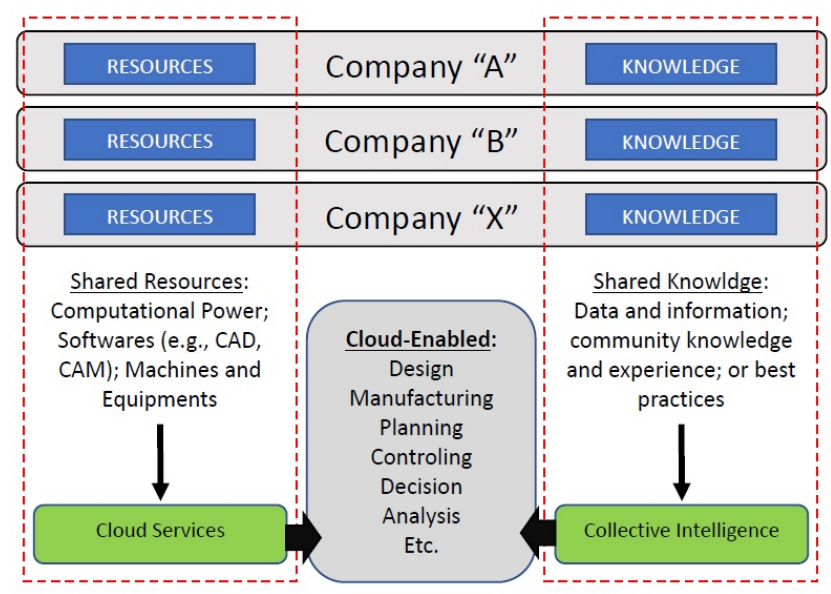

Fig. 3 Potentials of resources and knowledge sharing [14]

\section{Methodology}

In order to explore the researches on Cloud Manufacturing in a quantitative perspective, this article conducted a bibliometric study based on the appreciation of recorded information in the scientific literature. Subsequently, this analysis used tools and statistical techniques that resulted in indexes which enabled us to recognize correlations among the investigated publications [15]. Figure 4 presents the 5 steps conducted during this study.

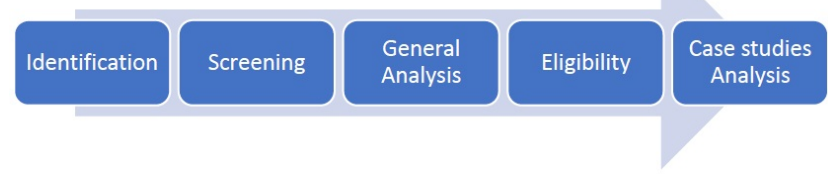

Fig. 4 The 5 steps applied in this bibliometric analysis [16]

The research and analysis methodology is illustrated as a flowchart in figure 5, organizing the 5 steps men-

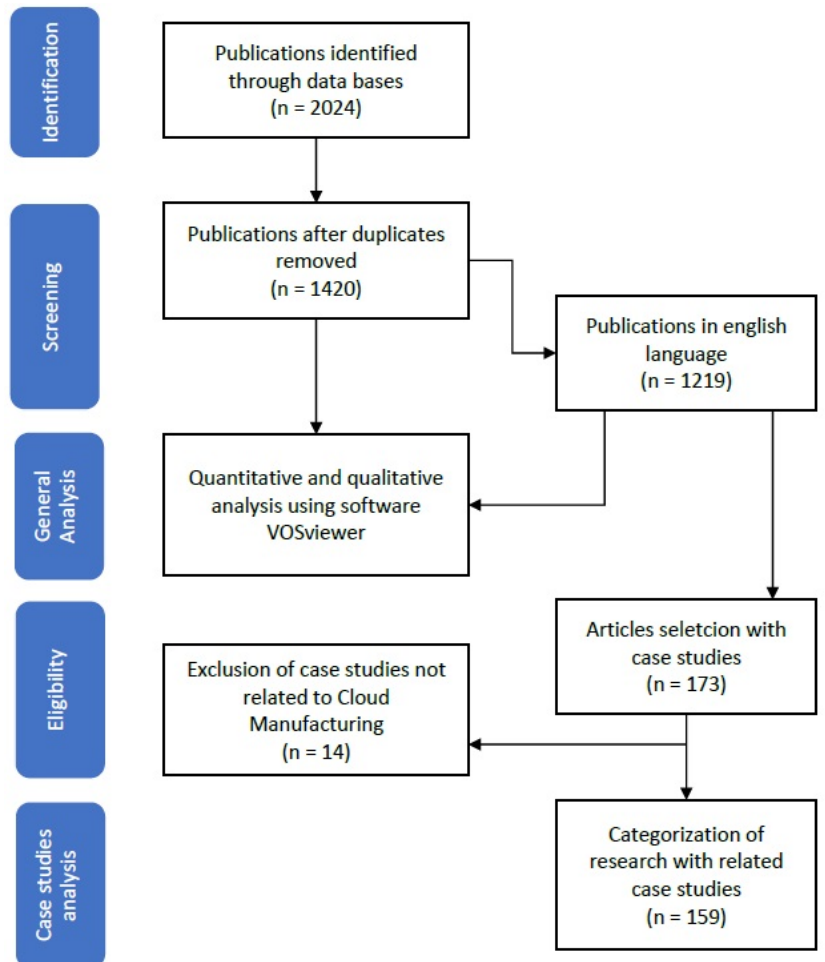

Fig. 5 Detailed flowchart of the research methodology [16]

tioned, with the registration of the quantity of articles detected in each step.

The 5 steps of the applied methodology are detailed as follows:

1. Identification of the scientifical publications related to Cloud Manufacturing, through researches in both Scopus and Web of Science data bases, using the search engine equations described on table 1. By doing so, this paper identified a total of 907 publications on Web of Science and 1,117 publications on Scopus.

2. Screening the publications using Microsoft Excel. By removing duplicates, this work found a total of 1,420 publications about Cloud Manufacturing to be analyzed. Still at the screening stage, the English language publications were separate (totalizing 1,219) in order to confront with other languages, at both the general and the case studies analysis steps.

3. General analysis over the 1,420 publications applying text mining techniques using a software called VOSviewer, developed by the University of Leiden, for construction of bibliometric clusters and maps, providing observation of the relationship of authors, keywords, citations, journals, countries and categorization of the most relevant terms [17].

4. Eligibility of the 159 articles containing applied case studies to quantify practical implementations about the Cloud Manufacturing emerging technology. In order to select the publications with meaningful cases, 
Table 1 Summary of the applied search engines [18]

\begin{tabular}{ll}
\hline \multicolumn{2}{c}{ Analysis of publications about Cloud Manufacturing } \\
\hline Data Bases & Web of Science and Scopus \\
\hline Total number of documents & 1,420 (907 from Web of Science and from 1,117 Scopus) \\
\hline General search engines terms & $\begin{array}{l}\text { ("cloud manufactur*" OR "cloud?based manufactur*" } \\
\text { OR "cloud?based design and manufactur*") }\end{array}$ \\
\hline $\begin{array}{l}\text { Specific search engine terms } \\
\text { (for case studies identification) }\end{array}$ & $\begin{array}{l}\text { (("cloud manufactur*" OR "cloud?based manufactur*" } \\
\text { OR "cloud?based design and manufactur*") AND ("case } \\
\text { stud*" OR "case analys*" OR "simulat*")) }\end{array}$ \\
\hline $\begin{array}{l}\text { Specific search filters } \\
\text { (for case studies) }\end{array}$ & $\begin{array}{l}\text { Publication Type: Article or Review } \\
\text { Language: English }\end{array}$ \\
\hline Softwares & VOSviewer and Excel \\
\hline Types of analysis & Literature review, quantitative and qualitative analysis \\
\hline
\end{tabular}

this paper searched the terms and applied the filters described on table 1.

5. Case studies analysis from both stratification and clusterization of the researches into the categories determined by VOSviewer, allowing us to identify the most relevant real-life applications of Cloud Manufacturing, as well as to recognize gaps and opportunities for future work.

\section{Results}

Over the 1,420 publications that arose from the screening process, this work built the graph presented in figure 6 that shows the overall trend of publications over time about Cloud Manufacturing, since the very first publication in 2010. It is possible to perceive an increase in the number of annual publications, confirming the growing relevance of this subject on both the academic and the industrial fields.
Figure 6 also presents the fitted trend line, generated using the statistical tool from Microsoft Excel, as the following linear equation.

$y=16.355 x+29.873 ; R^{2}=0.7616$

In order to avoid distortions in the trend equation, this exploration disregarded the 12 publications from January 2021, date when the bibliographic research was conducted.

Table 2 shows the countries with largest volume of publications in English language, indicating China as the leader, with an annual production greater than that of all other 14 countries together. China is also the country that presents the largest number of publications and of citations in any language.

Some countries stand out in the density of publications relative to their population, as New Zealand, Sweden and Finland, with publication rates considerably higher than those of the other countries on the list.

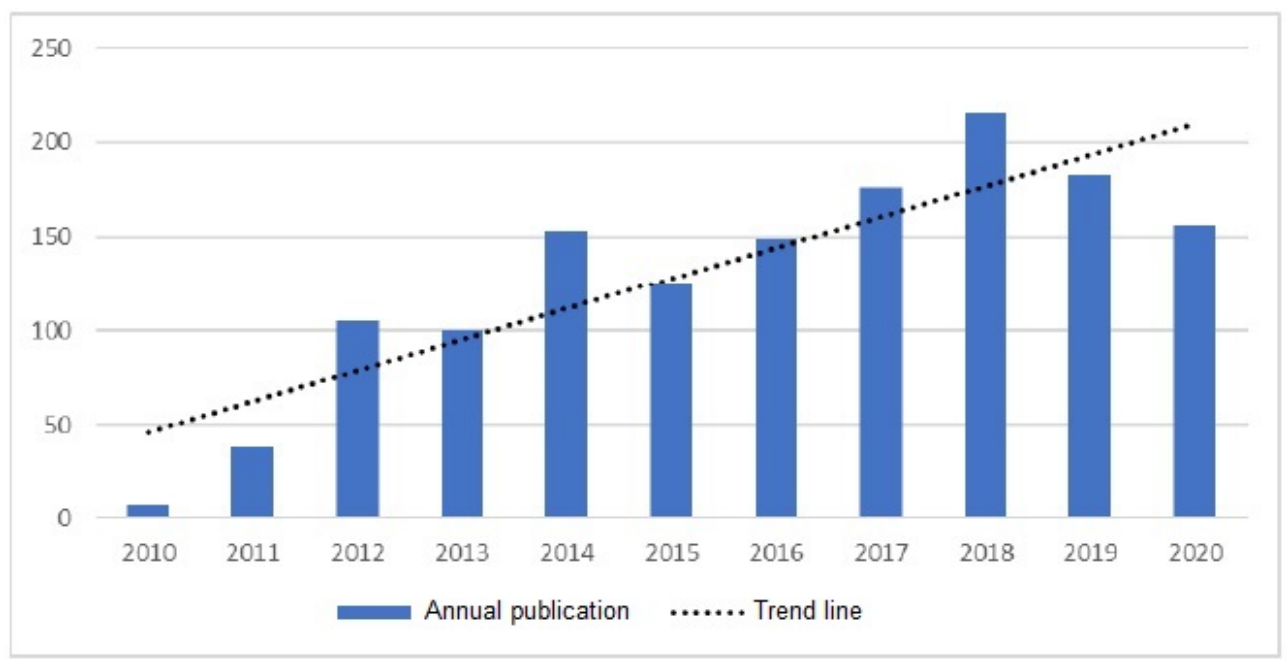

Fig. 6 Annual publications and trend about Cloud Manufacturing 
Table 2 Top 15 ranked countries with respect to the publications about Cloud Manufacturing

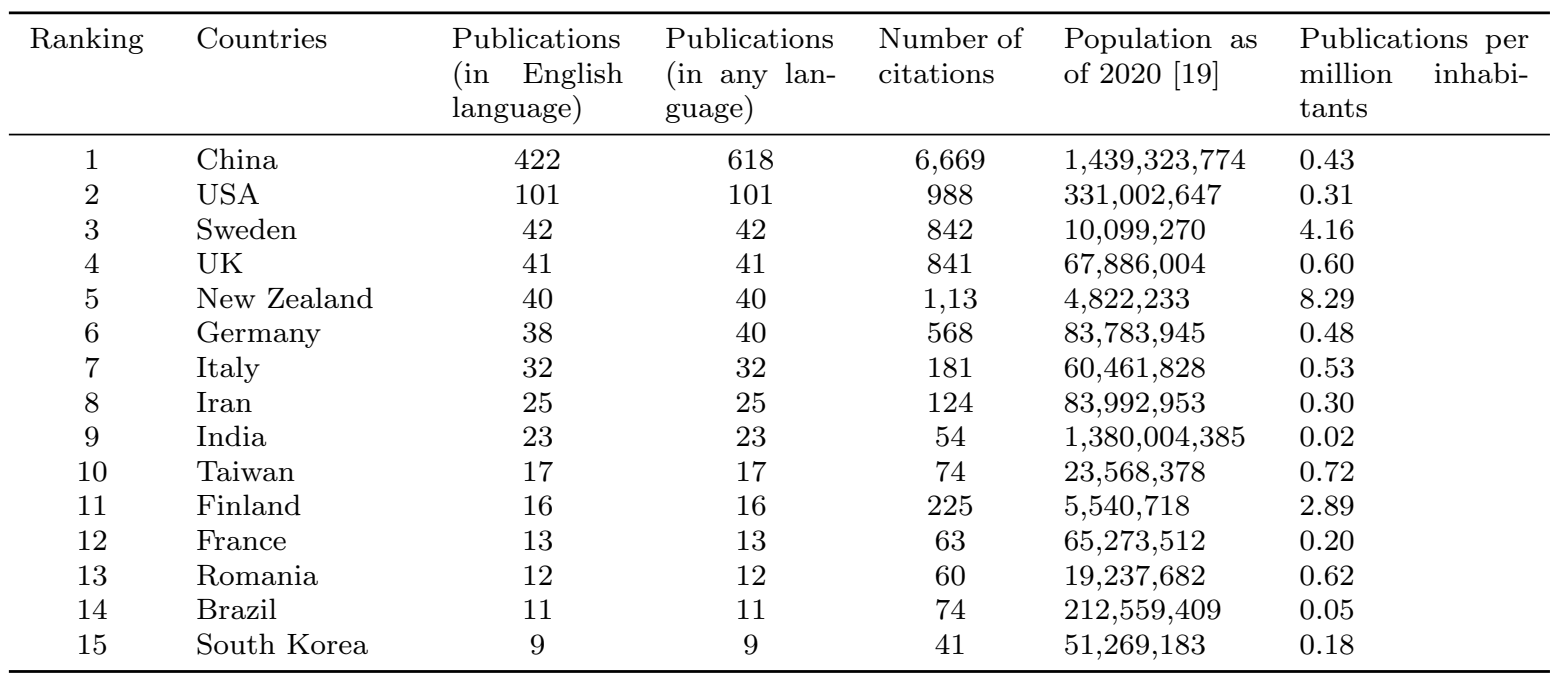

Table 3 Top 10 journals in number of publications

\begin{tabular}{|c|c|c|c|c|c|c|}
\hline Rank & Journal & $\begin{array}{l}\text { Country } \\
\text { gin }\end{array}$ & f ori- & Publications & Citations & Indexed Categories [20] \\
\hline 1 & $\begin{array}{l}\text { Computer Integrated Manufactur- } \\
\text { ing Systems, CIMS }\end{array}$ & China & & 123 & 3,233 & $\begin{array}{l}\text { Computer Science; Engineer- } \\
\text { ing }\end{array}$ \\
\hline 2 & $\begin{array}{l}\text { International Journal of Advanced } \\
\text { Manufacturing Technology }\end{array}$ & $\begin{array}{l}\text { United } \\
\text { dom }\end{array}$ & King- & 66 & 509 & $\begin{array}{l}\text { Computer Science; Engineer- } \\
\text { ing }\end{array}$ \\
\hline 3 & $\begin{array}{l}\text { International Journal of Computer } \\
\text { Integrated Manufacturing }\end{array}$ & $\begin{array}{l}\text { United } \\
\text { dom }\end{array}$ & King- & 41 & 472 & $\begin{array}{l}\text { Computer Science; Engineer- } \\
\text { ing }\end{array}$ \\
\hline 4 & $\begin{array}{l}\text { Robotics and Computer- } \\
\text { Integrated Manufacturing }\end{array}$ & $\begin{array}{l}\text { United } \\
\text { dom }\end{array}$ & King- & 36 & 645 & $\begin{array}{l}\text { Computer Science; Engineer- } \\
\text { ing; Mathematics }\end{array}$ \\
\hline 5 & $\begin{array}{l}\text { International Journal of Produc- } \\
\text { tion Research }\end{array}$ & $\begin{array}{l}\text { United } \\
\text { dom }\end{array}$ & King- & 29 & 251 & $\begin{array}{l}\text { Management Science, Opera- } \\
\text { tions Research; Industrial En- } \\
\text { gineering }\end{array}$ \\
\hline 6 & China Mechanical Engineering & \multicolumn{2}{|l|}{ China } & 28 & 107 & Mechanical Engineering \\
\hline 7 & $\begin{array}{l}\text { Journal of Intelligent Manufactur- } \\
\text { ing }\end{array}$ & \multicolumn{2}{|c|}{ Netherlands } & 21 & 487 & $\begin{array}{l}\text { Computer Science; Artificial } \\
\text { Intelligence; Industrial Engi- } \\
\text { neering; Manufacturing }\end{array}$ \\
\hline 8 & Journal of Manufacturing Systems & \multicolumn{2}{|c|}{ Netherlands } & 20 & 257 & $\begin{array}{l}\text { Computer Science; Engineer- } \\
\text { ing }\end{array}$ \\
\hline 9 & IEEE Access & \multicolumn{2}{|c|}{ United States } & 19 & 72 & $\begin{array}{l}\text { Computer Science; Engineer- } \\
\text { ing; Materials Science }\end{array}$ \\
\hline 10 & $\begin{array}{l}\text { Journal of Manufacturing Science } \\
\text { and Engineering-Transactions of } \\
\text { the ASME }\end{array}$ & \multicolumn{2}{|c|}{ United States } & 14 & 98 & $\begin{array}{l}\text { Computer Science; Engineer- } \\
\text { ing }\end{array}$ \\
\hline
\end{tabular}

Table 3 ranks the top 10 journals by total number of published papers. Their origin countries are China, United Kingdom, Netherlands and United States, with emphasis to the Chinese Computer Integrated Manufacturing Systems, CIMS, which boasts the largest number of publications, the highest number of citations, as well as the highest factor of citations per publication among the members of the list.

According to the indexed categories in Scimago [20], the most relevant journals are specialized on computer science, mechanical engineering, industrial engineering, mathematics, management science, operations research, manufacturing and artificial intelligence.

Table 4 shows the most influential articles on the subject of Cloud Manufacturing ranked by total citations, presenting keywords targeted for service-oriented business models, advanced manufacturing systems or platforms, internet of things (IoT), cloud computing, services optimization, real-time monitoring, big data, and distributed resources sharing. 
Table 4 Top ranked 16 most cited articles

\begin{tabular}{|c|c|c|c|c|c|}
\hline Rank & Citations & Title & Authors & Journal & Keywords \\
\hline 1 & 943 & $\begin{array}{l}\text { From cloud computing } \\
\text { to cloud manufacturing } \\
{[8]}\end{array}$ & $\begin{array}{l}\mathrm{Xu}, \quad \mathrm{X} \\
2012\end{array}$ & $\begin{array}{l}\text { Robot Comput } \\
\text { Integr Manuf }\end{array}$ & $\begin{array}{l}\text { Cloud computing; Cloud manufactur- } \\
\text { ing; Service-oriented business model }\end{array}$ \\
\hline 2 & 403 & $\begin{array}{l}\text { Cloud manufacturing: } \\
\text { a new manufacturing } \\
\text { paradigm }[10]\end{array}$ & $\begin{array}{l}\text { Zhang, } \\
\text { L; et al.; } \\
2014\end{array}$ & $\begin{array}{l}\text { Enterprise Infor- } \\
\text { mation Systems }\end{array}$ & $\begin{array}{l}\text { cloud manufacturing (CMfg); concept; } \\
\text { manufacturing cloud service; manufac- } \\
\text { turing cloud; cloud manufacturing ser- } \\
\text { vice platform }\end{array}$ \\
\hline 3 & 386 & $\begin{array}{l}\text { CCIoT-CMfg: Cloud } \\
\text { Computing and Internet } \\
\text { of Things-Based Cloud } \\
\text { Manufacturing Service } \\
\text { System [9] }\end{array}$ & $\begin{array}{l}\text { Tao, F; } \\
\text { et al.; } \\
2014\end{array}$ & $\begin{array}{lr}\text { IEEE } & \text { Trans- } \\
\text { actions } & \text { on } \\
\text { Industrial } & \text { Infor- } \\
\text { matics } & \end{array}$ & $\begin{array}{l}\text { Advanced manufacturing systems; } \\
\text { cloud computing; cloud manufacturin; } \\
\text { Internet of services; Internet of things } \\
\text { (IoT); Internet of users }\end{array}$ \\
\hline 4 & 356 & $\begin{array}{l}\text { IoT-Based Intelligent } \\
\text { Perception and Access } \\
\text { of Manufacturing Re- } \\
\text { source Toward Cloud } \\
\text { Manufacturing [12] }\end{array}$ & $\begin{array}{l}\text { Tao, F; } \\
\text { et al.; } \\
2014\end{array}$ & $\begin{array}{lr}\text { IEEE } & \text { Trans- } \\
\text { actions } & \text { on } \\
\text { Industrial } & \text { Infor- } \\
\text { matics } & \end{array}$ & $\begin{array}{l}\text { Access; cloud manufacturing; intelli- } \\
\text { gent perception; Internet of Things } \\
\text { (IoT); manufacturing resource; manu- } \\
\text { facturing service }\end{array}$ \\
\hline 5 & 337 & $\begin{array}{l}\text { Cloud manufacturing: a } \\
\text { computing and service- } \\
\text { oriented manufacturing } \\
\text { model }[13]\end{array}$ & $\begin{array}{l}\text { Tao, F.; } \\
\text { et al.; } \\
2011\end{array}$ & $\begin{array}{l}\text { Proc Inst Mech } \\
\text { Eng B J Eng } \\
\text { Manuf }\end{array}$ & $\begin{array}{l}\text { manufacturing system; cloud manu- } \\
\text { facturing; service-oriented manufactur- } \\
\text { ing; computing-oriented manufactur- } \\
\text { ing; cloud computing }\end{array}$ \\
\hline 6 & 332 & $\begin{array}{l}\text { Current status and } \\
\text { advancement of cyber- } \\
\text { physical systems in } \\
\text { manufacturing }[21]\end{array}$ & $\begin{array}{l}\text { Wang, } \\
\text { LH; et } \\
\text { al.; } 2015\end{array}$ & $\begin{array}{l}\text { Journal of } \\
\text { Manufacturing } \\
\text { Systems }\end{array}$ & \\
\hline 7 & 280 & $\begin{array}{l}\text { FC-PACO-RM: A Par- } \\
\text { allel Method for Service } \\
\text { Composition Optimal- } \\
\text { Selection in Cloud Man- } \\
\text { ufacturing System [22] }\end{array}$ & $\begin{array}{l}\text { Tao, F; } \\
\text { et al.; } \\
2012\end{array}$ & $\begin{array}{lr}\text { IEEE } & \text { Trans- } \\
\text { actions } & \text { on } \\
\text { Industrial } & \text { Infor- } \\
\text { matics } & \end{array}$ & $\begin{array}{l}\text { Cloud computing; cloud manufactur- } \\
\text { ing; full connection; parallel adaptive } \\
\text { chaos optimization; reflex migration; } \\
\text { service composition optimal-selection }\end{array}$ \\
\hline 8 & 199 & $\begin{array}{l}\text { An interoperable solu- } \\
\text { tion for Cloud manufac- } \\
\text { turing }[11]\end{array}$ & $\begin{array}{l}\text { Wang, } \\
\text { XV; Xu, } \\
\text { XW; } \\
2013\end{array}$ & $\begin{array}{l}\text { Robot Comput } \\
\text { Integr Manuf }\end{array}$ & $\begin{array}{l}\text { Cloud; Cloud computing; Cloud man- } \\
\text { ufacturing; Service-oriented architec- } \\
\text { ture; STEP }\end{array}$ \\
\hline 9 & 193 & $\begin{array}{l}\text { Big Data and virtualiza- } \\
\text { tion for manufacturing } \\
\text { cyber-physical systems: } \\
\text { A survey of the current } \\
\text { status and future out- } \\
\text { look [23] }\end{array}$ & $\begin{array}{l}\text { Babiceanu, } \\
\text { RF; } \\
\text { Seker, } \\
\text { R; } 2016\end{array}$ & $\begin{array}{l}\text { Computers in In- } \\
\text { dustry }\end{array}$ & $\begin{array}{l}\text { Sensor-based real-time monitoring; Big } \\
\text { Data; Internet of things; Cloud com- } \\
\text { puting; Manufacturing cyber-physical } \\
\text { systems }\end{array}$ \\
\hline 10 & 173 & $\begin{array}{l}\text { Cloud-enabled progno- } \\
\text { sis for manufacturing } \\
{[14]}\end{array}$ & $\begin{array}{l}\text { Gao, } R ; \\
\text { et al.; } \\
2015\end{array}$ & $\begin{array}{l}\text { CIRP Ann } \\
\text { Manuf Technol }\end{array}$ & $\begin{array}{l}\text { Predictive model; Condition monitor- } \\
\text { ing; Cloud manufacturing }\end{array}$ \\
\hline 11 & 164 & $\begin{array}{l}\text { Advanced manufactur- } \\
\text { ing systems: socializa- } \\
\text { tion characteristics and } \\
\text { trends }[24]\end{array}$ & $\begin{array}{l}\text { Tao, F; } \\
\text { et al.; } \\
2017\end{array}$ & $\begin{array}{l}\text { Journal of Intel- } \\
\text { ligent Manufac- } \\
\text { turing }\end{array}$ & $\begin{array}{l}\text { Advanced manufacturing system; So- } \\
\text { cialization; Service; Resource shar- } \\
\text { ing; Value creation; User participation; } \\
\text { Cloud manufacturing }\end{array}$ \\
\hline 12 & 133 & $\begin{array}{l}\text { Cloud manufacturing: } \\
\text { key characteristics and } \\
\text { applications }[25]\end{array}$ & $\begin{array}{l}\text { Ren, L; } \\
\text { et al.; } \\
2017\end{array}$ & $\begin{array}{l}\text { Int. J. Comput. } \\
\text { Integr. Manuf. }\end{array}$ & $\begin{array}{l}\text { cloud manufacturing; cloud comput- } \\
\text { ing; Internet of Things; cloud business } \\
\text { model }\end{array}$ \\
\hline 13 & 132 & $\begin{array}{l}\text { A state-of-the-art sur- } \\
\text { vey of cloud manufac- } \\
\text { turing }[26]\end{array}$ & $\begin{array}{l}\mathrm{He}, \mathrm{W} \\
\mathrm{Xu}, \mathrm{LD} \\
2015\end{array}$ & $\begin{array}{l}\text { Int. J. Comput. } \\
\text { Integr. Manuf. }\end{array}$ & $\begin{array}{l}\text { distributed resources; cloud manufac- } \\
\text { turing; resource integration; enterprise } \\
\text { services; service management; Internet } \\
\text { of Things (IoT); cloud computing }\end{array}$ \\
\hline 14 & 128 & $\begin{array}{l}\text { IoT-based real-time pro- } \\
\text { duction logistics syn- } \\
\text { chronization system un- } \\
\text { der smart cloud manu- } \\
\text { facturing }[27]\end{array}$ & $\begin{array}{l}\text { Qu, T; } \\
\text { et al.; } \\
2016\end{array}$ & $\begin{array}{l}\text { Int. J. Adv. } \\
\text { Manuf. Technol. }\end{array}$ & $\begin{array}{l}\text { Cloud manufacturing; Internet of } \\
\text { things; Production logistic; Dynamic } \\
\text { synchronization }\end{array}$ \\
\hline 15 & 125 & $\begin{array}{l}\text { Cloud manufacturing: } \\
\text { from concept to practice } \\
{[28]}\end{array}$ & $\begin{array}{l}\text { Ren, L; } \\
\text { et al.; } \\
2015\end{array}$ & $\begin{array}{l}\text { Enterprise Infor- } \\
\text { mation Systems }\end{array}$ & $\begin{array}{l}\text { cloud manufacturing; public cloud; } \\
\text { cloud computing; service-oriented busi- } \\
\text { ness model; cloud platform; informa- } \\
\text { tion systems }\end{array}$ \\
\hline 16 & 118 & $\begin{array}{l}\text { Cloud manufacturing } \\
\text { service platform for } \\
\text { small- and medium- } \\
\text { sized enterprises [29] }\end{array}$ & $\begin{array}{l}\text { Huang, } \\
\text { BQ; et } \\
\text { al.; } 2013\end{array}$ & $\begin{array}{l}\text { Int. J. Adv. } \\
\text { Manuf. Technol. }\end{array}$ & $\begin{array}{l}\text { Cloud manufacturing; Small- and } \\
\text { medium sized enterprise (SME); Cloud } \\
\text { manufacturing service platform }\end{array}$ \\
\hline
\end{tabular}




\subsection{Clustering Analysis}

Using the software VOSviewer, this study built figure 7 that reveals the keywords co-occurrences map, determinated by the documents where they are found together. Both authors keywords, and other relevant words frequently used in titles and abstracts were considered.

Every keyword is represented by a circle, whose size is proportional to its frequency of occurrence. The positions indicate the relationship between two different keywords, and the lines signify connectivity and association of concepts.

Among the 1,420 analyzed papers, this step found a total of 4,487 keywords, of which 104 met the requirement of having at least 10 occurrences each, being then applied in the mapping process.

Figure 7 shows the characterization of the four cooccurrences clusters:
- the first cluster (colored red) is defined as "Collaborative networks of manufacturing resources and services", once the most frequent terms refers to manufacture, manufacturing resources and services, distributed systems architecture, and networked manufacturing environments through web-based services;

- the second (green) is defined as "Industry 4.0 and cloud computing systems", once the most frequent keywords are related to computer-aided manufacturing processes in industry 4.0. This cluster encompasses the whole product lifecycle within industry 4.0, including cloud computing technologies, internet of things (IoT) and additive manufacturing;

- the third (blue) is defined as "Big data reliable management in cyber-physical systems, risks and challenges", once the main occurrences are related to big data in cyber-physical systems, processes integration,

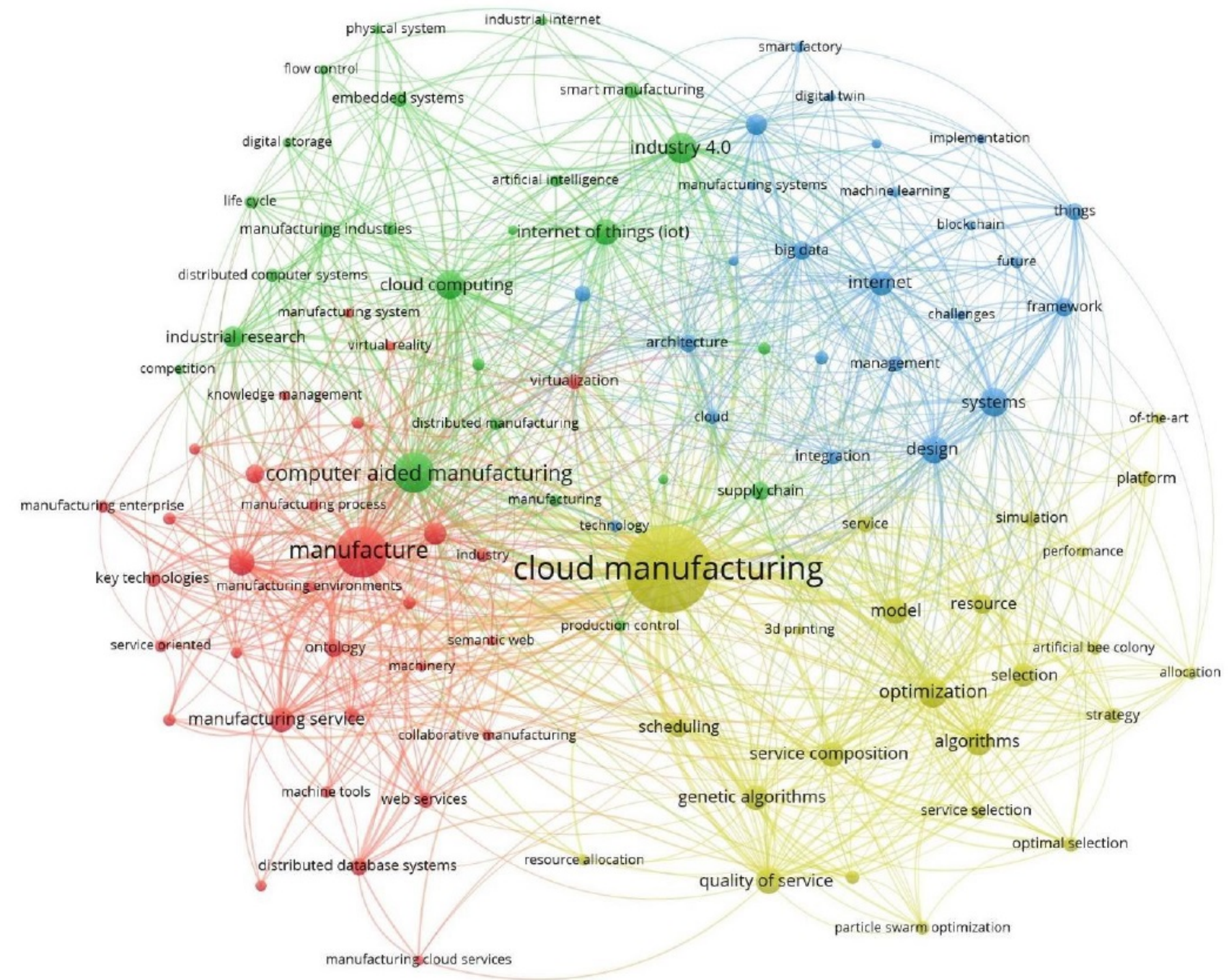

Fig. 7 Keywords co-occurrence map 
Table 5 Characterization and composition of the keywords clusters

\begin{tabular}{lcl}
\hline Clusters & Itens & Keywords (Occurrences) \\
\hline (1) Collaborative net- & 31 & manufacture (267); manufacturing resource (69); manufacturing service (64); cloud \\
works of manufactur- & & services (51); ontology (37); computer architecture (34); distributed database sys- \\
ing resources and ser- & tems (30); web services (25); key technologies (23); virtualization (23); semantics \\
vices & $(22)$; industry (20); manufacturing environments (18); service oriented (18); man- \\
(red) & ufacturing enterprise (17); manufacturing process (17); networked-manufacturing \\
& $(17) ;$ service platforms (17); service-oriented architecture (17); manufacturing ca- \\
& pability (16); knowledge based systems (14); collaborative manufacturing (13); in- \\
& formation services (13); machine tools (13); mathematical models (13); machinery \\
& $(12) ;$ semantic web (12); knowledge management (11); virtual reality (11); manu- \\
& facturing cloud services (10); manufacturing system (10).
\end{tabular}

(2) Industry 4.0 and 25 computer aided manufacturing (164); industry 4.0 (94); cloud computing (84); cloud computing sys- internet of things (iot) (70); industrial research (46); supply chain (35); smart tems manufacturing (27); manufacturing industries (24); embedded systems (23); distributed computer systems (20); product design (19); distributed manufacturing (18); life cycle (18); additive manufacturing (17); decision making (17); manufacturing (17); artificial intelligence (16); production control (14); competition (11); mass customization (11); cyber physical system (10); digital storage (10); flow control (10); industrial internet (10); production planning (10).

(3) Big data reliable 24 systems (80); design (76); internet (63); cyber-physical systems (45); big data (37); management in cyber- $\quad$ framework (36); architecture (33); things (30); management (28); intelligent manuphysical systems, risks facturing (26); integration (25); interoperability (21); cloud (19); technology (18); and challenges future (16); smart factory (13); blockchain (11); machine learning (11); manu(blue) facturing systems (11); challenges (10); digital twin (10); implementation (10); industrial internet of things (10); resource virtualization (10).

$\begin{array}{lll}\text { (4) Optimization of } 24 & \text { cloud manufacturing (751); optimization (93); algorithms (77); model (69); quality } \\ \text { manufacturing pro- } & & \text { of service (69); genetic algorithms (61); service composition (60); selection (55); } \\ \text { cesses } & \text { scheduling (43); resource (39); service (28); strategy (27); platform (26); optimal } \\ \text { (yellow) } & \text { selection (23); service selection (23); simulation (22); multi-objective optimization } \\ & \text { (21); particle swarm optimization (19); artificial bee colony (16); allocation (15); } \\ & \text { of-the-art (13); performance (13); resource allocation (13); 3d printing (10). }\end{array}$

interoperability, blockchain and implementation challenges; and finally

- the fourth cluster (yellow) is defined as "Optimization of manufacturing processes", once the most frequent keywords are related to optimization algorithms and models of cloud manufacturing, as well as to optimal selection and allocation of resources and services.

The elements of each cluster are shown in table 5 .

\subsection{Case Studies Analysis}

The case study approach allows researchers to explore and understand complex problems in real-life applications and, therefore, is recognized by its capacity to generate value to the industrial and academic sectors.

In order to assess practical applications in Cloud Manufacturing, this investigation performed the eligibility step, applying the previously-described search filters and terms showed in table 1 , resulting in the selection of 159 academic papers with applied case studies out of 1,219 publications in English, as shown in figure 8.
Afterwards, all titles, abstracts and keywords of the 159 articles were analyzed, enabling the categorization of their case studies approaches within the four preestablished co-occurrences clusters. Figure 9 presents the final stratification by cluster with the number of related articles.

- Optimization of manufacturing processes (69 articles): This cluster contains all case studies which presented platform models or algorithms to generate optimized solutions on: (i) production planning [30], (ii) scheduling and selection of manufacturing resources or

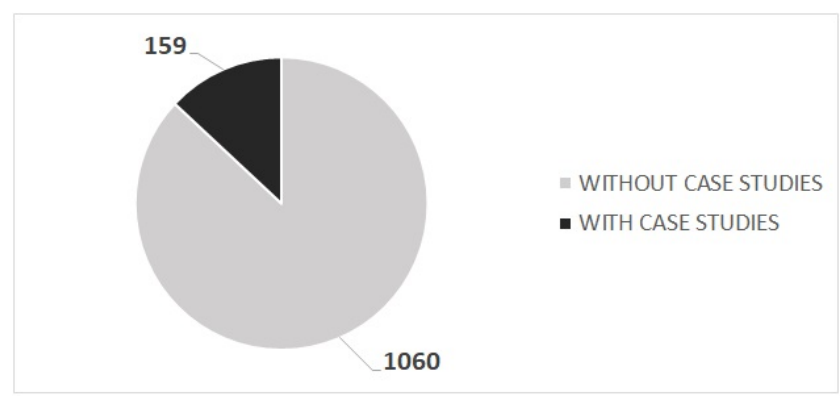

Fig. 8 Publications in English language, with and without case studies 


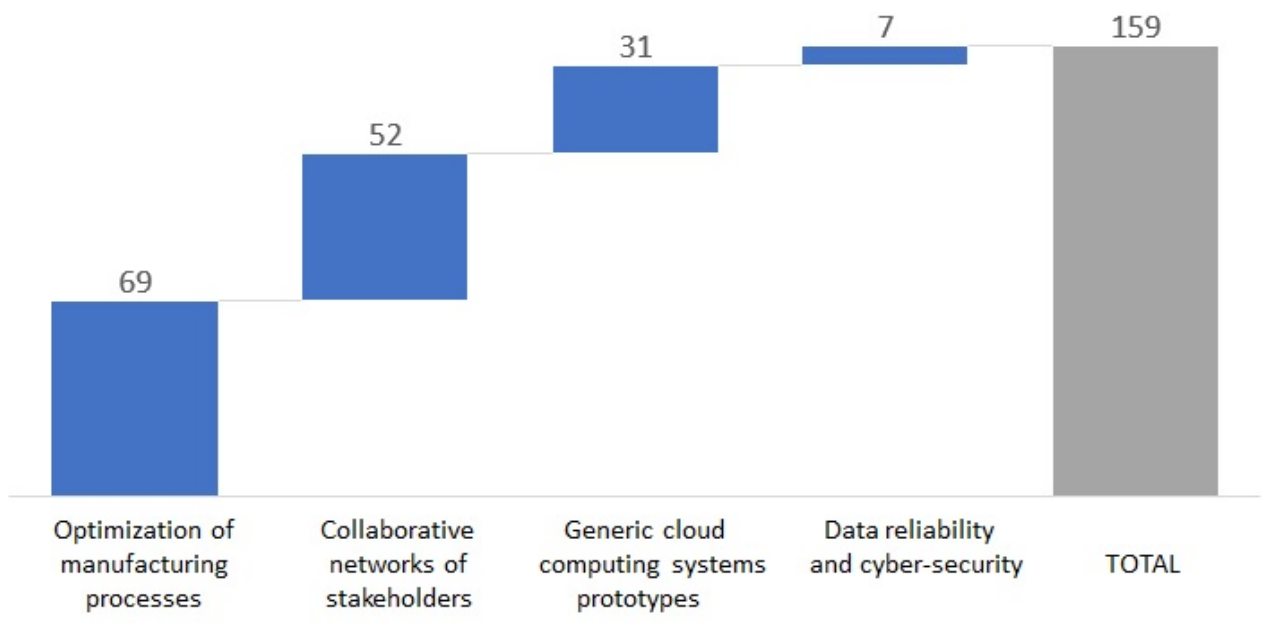

Fig. 9 Case studies classified by their approach

services [31][32], (iii) reduction of operational and/or logistic total time [33], (iv) cost reduction, (v) maximization of utilization rates [34], (vi) balancing the allocation levels of machines and tools [35], (vii) minimization of industrial resources consumption by controlling process parameters [36], (viii) energy efficiency improvements [37], (ix) productivity increase, (x) task prioritization [38], as well as other strategies aiming at improving factory performance when compared to traditional approaches.

- Collaborative networks of manufacturing resources and services (52 articles): This cluster contains the models developed as tools to approach the relationship between stakeholders, aiming at assessing: (i) synergy of the manufacturing services and enterprises networks [39], (ii) integration of operations, information, knowledge and efforts between players [40][41], (iii) the sharing of resources capabilities [42], (iv) collaborative troubleshooting process involving multiple companies [43], (v) the dynamic decision-making process made by customers to meet their customized demands in production networks with high levels of flexibility and responsiveness [44], (vi) procedures that increase integration of capabilities in remanufacturing, recycling and recovery [45], as well as other strategies that might increase competitiveness throughout the manufacturing supply chain.

- Industry 4.0 and cloud computing systems (31 articles): This cluster contains studies about experimental prototypes, platforms and/or applications of practical demonstrations that aimed at illustrating the applicability of Cloud Manufacturing in a generic point of view. Such cases must either: (i) present the application of emerging technologies as additive manufacturing (3D printing) [46], internet of things (IoT) [47] and other human-machine interaction approaches in advanced manufacturing of industry 4.0, (ii) test the functionality of prototypes, comparing them to other models in order to validate benefits and disadvantages of adopting Cloud Manufacturing networks and systems [23], or (iii) highlight implementation challenges and requirements to achieve success in the adoption of virtual manufacturing [11][29].

- Data reliability, cyber-security, risks and challenges (7 articles): This cluster contains all studies about: (i) modeling and simulating architectures that focus both on maximizing transparent data analysis in real-time and on improving cyber-security [48], (ii) using consensus and Proof-of-Authority (PoA) resources to improve the reliability of transaction records between various agents [49], (iii) the application of blockchain technology [50], and (iv) the evaluation of risks or potential failures in transmission of data and/or communication between companies [51].

The great majority of the case studies assessed by this work utilized various computational models, cyberphysical concepts, IT services, and mathematical methods to support decision making and to create collaborative networks. The most common approaches include Genetic Algorithm [34], Ant Colony [52], Bee Colony [53], Particle Swarm optimization [50], K-Nearest Neighbors (KNN) [49], Chaos Theory [54], Fuzzy logic [55], Game Theory [42], TOPSIS [35], Kano model [56], Artificial Neural Networks [57], Grey Wolf optimizer [37], AHP (Analytic hierarchy process) [52], Blockchain Ethereum [49], and multi-agent systems [58]. Besides, the cases used several simulation softwares, such as Simio [59], FlexSim [60], CloudSim [61], SDMSim [62], MathLab [63], Windows Azure [64], ZigBee [65] and other webbased applications. 


\section{Conclusions}

From the bibliometric analysis this research conducted on the emerging technology of Cloud Manufacturing, it was possible to map the current literature conditions and to verify that, since 2010, when the first studies appeared, there has been a growing trend in annual publications, suggesting an increasing interest about the subject.

The results obtained reveal that researchers from China are those who generate the highest volume of publications, even when only English language articles are taken into account. Another interesting evidence concerns countries such as New Zealand, Sweden and Finland, which presented considerably high publications rates relative to their populations.

It was not only possible to identify the most relevant journals, with the top editions coming from USA, United Kingdom, Netherlands and China, but also a list of the top-ranked articles in terms of influence relative to their number of citations. Those lists can be useful references for researchers interested in the literature about Cloud Manufacturing.

By using the software VOSviewer, this paper elaborated a visualization of bibliometric maps and created four clusters of keywords co-occurrences: "collaborative networks of manufacturing resources and services"; "industry 4.0 and cloud computing systems"; "big data reliability, cyber-security, risks and challenges"; and "optimization of manufacturing processes".

In order to understand the current situation about real-life applications already developed and studied in Cloud Manufacturing, this investigation selected and analyzed all 159 articles written in English that contained case studies. All of them were assessed and categorized according to the four previously-cited clusters. It became evident that, while most of the models laid within three of the four clusters - "optimization of manufacturing processes", "collaborative networks of manufacturing resources and services", and "industry 4.0 and cloud computing systems" -, only a few of them were related to "big data reliability, cyber-security, risks and challenges".

The lack of publications about data and risk management in Cloud Manufacturing reveal a potential research gap. The absence of depth in such an important matter is also an opportunity for development of future works and researches on the application of technologies for the evolution of cyber-security that could potentially reduce breaches or vulnerabilities in cloud manufacturing chains, and contribute to technological advancements in a new, yet underexplored, field.
Acknowledgements We thank CENPRO (Industrial Engineering Research Center - FCA/UNICAMP) for all the intellectual contribution and the technical support that added great value to this work.

\section{Declarations}

Funding: No funding was received for conducting this study.

Conflicts of interest: The authors declare that they have no conflicts of interest.

Availability of data and material (data transparency): Not applicable.

Code availability (software application or custom code): Not applicable.

\section{References}

1. Benitez, G. B., Lima, M. J. D. R. F., Lerman, L. V., Frank, A. G. (2019). Understanding Industry 4.0: Definitions and insights from a cognitive map analysis. Brazilian Journal of Operations Production Management [recurso eletrônico]. Rio de Janeiro, RJ. Vol. 16, no. 2 (June 2019), p. 192-200. https://doi.org/10.14488/BJOPM.2019.v16.n2.a3

2. Charro, A., Schaefer, D. (2018). Cloud Manufacturing as a new type of Product-Service System. International Journal of Computer Integrated Manufacturing, 31(10), 1018-1033. https://doi.org/10.1080/0951192x.2018.1493228

3. Wu, D., Greer, M. J., Rosen, D. W., Schaefer, D. (2013). Cloud manufacturing: Strategic vision and state-of-theart. Journal of Manufacturing Systems, 32(4), 564-579. https://doi.org/10.1016/j.jmsy.2013.04.008

4. da Silva Bonifácio, E., Singnorete, N. A., de Arruda Ignácio, P. S., Stoco, W. H. (2020). Integração entre fornecedor e varejista para gestão de estoque através da tecnologia cloud-uma proposta para melhorar a performance do fluxo de informações. Brazilian Journal of Business, 2(3), 2049-2056. https://doi.org/10.34140/bjbv2n3-014

5. Li, B. H., Zhang, L., Wang, S. L., Tao, F., et al. (2010). Cloud manufacturing: a new service-oriented networked manufacturing model. Computer integrated manufacturing systems, 16(1), 1-7.

6. Marston, S., Li, Z., Bandyopadhyay, S., Zhang, J., Ghalsasi, A. (2011). Cloud computing-The business perspective. Decision support systems, 51(1), 176-189. https://doi.org/10.1109/HICSS.2011.102

7. Sala-Zárate, M., Colombo-Mendoza, L. (2012). Cloud computing: a review of PAAS, IAAS, SAAS services and providers. Lámpsakos, (7), 47-57. https://doi.org/10.21501/21454086.844

8. Xu, X. (2012). From cloud computing to cloud manufacturing. Robotics and computer-integrated manufacturing, 28(1), 75-86. https://doi.org/10.1016/j.rcim.2011.07.002

9. Tao, F., Cheng, Y., Da Xu, L., Zhang, L., Li, B. H. (2014). CCIoT-CMfg: cloud computing and internet of things-based cloud manufacturing service system. IEEE Transactions on industrial informatics, 10(2), 1435-1442. https://doi.org/10.1109/TII.2014.2306383

10. Zhang, L., Luo, Y., Tao, F., Li, B. H., et al. (2014). Cloud manufacturing: a new manufacturing paradigm. Enterprise Information Systems, 8(2), 167-187. https://doi.org/10.1080/17517575.2012.683812 
11. Wang, X. V., Xu, X. W. (2013). An interoperable solution for cloud manufacturing. Robotics and computer-integrated manufacturing, 29(4), 232-247. https://doi.org/10.1016/j.rcim.2013.01.005

12. Tao, F., Zuo, Y., Da Xu, L., Zhang, L. (2014). IoT-based intelligent perception and access of manufacturing resource toward cloud manufacturing. IEEE Transactions on Industrial Informatics, 10(2), 1547-1557. https://doi.org/10.1109/TII.2014.2306397

13. Tao, F., Zhang, L., Venkatesh, V. C., Luo, Y., Cheng, Y. (2011). Cloud manufacturing: a computing and service-oriented manufacturing model. Proceedings of the Institution of Mechanical Engineers, Part B: Journal of Engineering Manufacture, 225(10), 1969-1976. https://doi.org/10.1177/0954405411405575

14. Gao, R., Wang, L., Teti, R., Dornfeld, D., Kumara, S., Mori, M., Helu, M. (2015). Cloud-enabled prognosis for manufacturing. CIRP annals, 64(2), 749-772. https://doi.org/10.1016/j.cirp.2015.05.011

15. Camatti, J. A., Arthus, M. G., Silva, A. L., Cesar, F. I. G., Ignacio, P. S. A. (2016). "O impacto da produção enxuta no desempenho dos trabalhadores: uma análise bibliométrica", VI Congresso Brasileiro de Engenharia de Produção, APREPRO, Anais VI 2016.

16. Yang, G. Y., Wang, L. Q., Ren, J., Zhang, Y., et al. (2015). Evidence base of clinical studies on Tai Chi: a bibliometric analysis. PloS one, 10(3), e0120655. https://doi.org/10.1371/journal.pone.0120655

17. Van Eck, N. J., Waltman, L. (2010). Software survey: VOSviewer, a computer program for bibliometric mapping. scientometrics, 84(2), 523-538. https://doi.org/10.1007/s11192-009-0146-3

18. De Oliveira, R. I., Sousa, S. O., De Campos, F. C. (2019). Lean manufacturing implementation: bibliometric analysis 2007-2018. The International Journal of Advanced Manufacturing Technology, 101(1), https://doi.org/979988. 10.1007/s00170-018-2965-y

19. Pyramids Population of the World (2020), URL: https://www.populationpyramid.net/, Accessed: February 9, 2021 .

20. Scimago Institutions Ranking (2020), Journal Rankings, powered by SCOPUS, URL: https://www.scimagojr.com/journalrank.php, Accessed: February 10, 2021.

21. Wang, L., Törngren, M., Onori, M. (2015). Current status and advancement of cyber-physical systems in manufacturing. Journal of Manufacturing Systems, 37, 517-527. https://doi.org/10.1016/j.jmsy.2015.04.008

22. Tao, F., LaiLi, Y., Xu, L., Zhang, L. (2012). FCPACO-RM: a parallel method for service composition optimal-selection in cloud manufacturing system. IEEE Transactions on Industrial Informatics, 9(4), 2023-2033. https://doi.org/10.1109/TII.2012.2232936

23. Babiceanu, R. F., Seker, R. (2016). Big Data and virtualization for manufacturing cyber-physical systems: A survey of the current status and future outlook. Computers in Industry, 81, 128-137. https://doi.org/10.1016/j.compind.2016.02.004

24. Tao, F., Cheng, Y., Zhang, L., Nee, A. Y. (2017). Advanced manufacturing systems: socialization characteristics and trends. Journal of Intelligent Manufacturing, 28(5), 1079-1094. https://doi.org/10.1007/s10845-015-1042-8

25. Ren, L., Zhang, L., Wang, L., Tao, F., Chai, X. (2017). Cloud manufacturing: key characteristics and applications. International journal of computer integrated manufacturing, 30(6), 501-515. https://doi.org/10.1080/0951192X.2014.902105
26. He, W., Xu, L. (2015). A state-of-the-art survey of cloud manufacturing. International Journal of Computer Integrated Manufacturing, 28(3), 239-250. https://doi.org/10.1080/0951192X.2013.874595

27. Qu, T., Lei, S. P., Wang, Z. Z., et al. (2016). IoT-based real-time production logistics synchronization system under smart cloud manufacturing. The International Journal of Advanced Manufacturing Technology, 84(1-4), 147-164. https://doi.org/10.1007/s00170-015-7220-1

28. Ren, L., Zhang, L., Tao, F., et al. (2015). Cloud manufacturing: from concept to practice. Enterprise Information Systems, 9(2), 186-209. https://doi.org/10.1080/17517575.2013.839055

29. Huang, B., Li, C., Yin, C., Zhao, X. (2013). Cloud manufacturing service platform for small-and mediumsized enterprises. The International Journal of Advanced Manufacturing Technology, 65(9-12), 1261-1272. https://doi.org/10.1007/s00170-012-4255-4

30. Valizadeh, S., Fatahi Valilai, O., Houshmand, M. (2020). Flexible flow line scheduling considering machine eligibility in a digital dental laboratory. International Journal of Production Research, 58(21), 6513-6531. https://doi.org/10.1080/00207543.2019.1683247

31. Simeone, A., Zeng, Y., Caggiano, A. (2020). Intelligent decision-making support system for manufacturing solution recommendation in a cloud framework. The International Journal of Advanced Manufacturing Technology, 116. https://doi.org/10.1007/s00170-020-06389-1

32. Wang, L., Guo, S., Li, X., Du, B., Xu, W. (2018). Distributed manufacturing resource selection strategy in cloud manufacturing. The International Journal of Advanced Manufacturing Technology, 94(9), 3375-3388. https://doi.org/10.1007/s00170-016-9866-8

33. Liu, Y., Xu, X., Zhang, L., Tao, F. (2016). An extensible model for multitask-oriented service composition and scheduling in cloud manufacturing. Journal of Computing and Information Science in Engineering, 16(4). https://doi.org/10.1115/1.4034186

34. Simeone, A., Deng, B., Caggiano, A. (2020). Resource efficiency enhancement in sheet metal cutting industrial networks through cloud manufacturing. The International Journal of Advanced Manufacturing Technology, 107(3), 1345-1365. https://doi.org/10.1007/s00170-020-05083-6

35. Li, X., Yin, C., Liu, F. (2017). A trust estimation method of machine tool resources in the cloud environment. Journal of Statistical Computation and Simulation, 87(13), 25722580. https://doi.org/10.1080/00949655.2017.1349130

36. Mourtzis, D., Vlachou, E., Milas, N., Tapoglou, N., Mehnen, J. (2019). A cloud-based, knowledgeenriched framework for increasing machining efficiency based on machine tool monitoring. Proceedings of the Institution of Mechanical Engineers, Part B: Journal of Engineering Manufacture, 233(1), 278-292. https://doi.org/10.1177/0954405417716727

37. Yang, Y., Yang, B., Wang, S., Liu, W., Jin, T. (2019). An improved grey wolf optimizer algorithm for energyaware service composition in cloud manufacturing. The International Journal of Advanced Manufacturing Technology, 105(7), 3079-3091. https://doi.org/10.1007/s00170019-04449-9

38. Zhou, L., Zhang, L., Zhao, C., Laili, Y., Xu, L. (2018). Diverse task scheduling for individualized requirements in cloud manufacturing. Enterprise Information Systems, 12(3), 300-318. http://dx.doi.org/10.1080/17517575.2017.1364428

39. Ren, M., Ren, L., Jain, H. (2018). Manufacturing service composition model based on synergy effect: a social network 
analysis approach. Applied Soft Computing, 70, 288-300. https://doi.org/10.1016/j.asoc.2018.05.039

40. Zhao, C., Luo, X., Zhang, L. (2020). Modeling of service agents for simulation in cloud manufacturing. Robotics and Computer-Integrated Manufacturing, 64, 101910. https://doi.org/10.1016/j.rcim.2019.101910

41. Li, B., Liu, S., Guo, Y., Du, Z., Lei, Z., Ding, Z. (2020). Multi-Core and Cross-Chain Evaluation Method Based on Multi-Core Mesh Collaboration Relationship. IEEE Access, 8, 151829-151846. https://doi.org/10.1109/ACCESS.2020.3014212

42. Argoneto, P., Renna, P. (2016). Supporting capacity sharing in the cloud manufacturing environment based on game theory and fuzzy logic. Enterprise Information Systems, 10(2), 193-210. https://doi.org/10.1080/17517575.2014.928950

43. Cai, X., Li, W., He, F., Li, X. (2015). Customized encryption of computer aided design models for collaboration in cloud manufacturing environment. Journal of Manufacturing Science and Engineering, 137(4). https://doi.org/10.1115/1.4030592

44. Cheng, Y., Tao, F., Zhao, D., Zhang, L. (2017). Modeling of manufacturing service supply-demand matching hypernetwork in service-oriented manufacturing systems. Robotics and Computer-Integrated Manufacturing, 45, 5972. https://doi.org/10.1016/j.rcim.2016.05.007

45. Wang, X. V., Wang, L. (2017). A cloud-based production system for information and service integration: an internet of things case study on waste electronics. Enterprise Information Systems, 11(7), 952-968. https://doi.org/10.1080/17517575.2016.1215539

46. Zhang, C., Zhao, F., Wang, Z. (2019). Modeling of Cloud 3D Printing Service Hyper-Network in Service-Oriented Manufacturing Systems. IEEE Access, 8, 16225-16235. https://doi.org/10.1109/ACCESS.2019.2962248

47. Rasouli, M. R. (2020). An architecture for IoT-enabled intelligent process-aware cloud production platform: a case study in a networked cloud clinical laboratory. International Journal of Production Research, 58(12), 3765-3780. https://doi.org/10.1080/00207543.2019.1634847

48. Li, Z., Barenji, A. V., Huang, G. Q. (2018). Toward a blockchain cloud manufacturing system as a peer to peer distributed network platform. Robotics and computer-integrated manufacturing, 54, 133-144. https://doi.org/10.1016/j.rcim.2018.05.011

49. Zhu, X., Shi, J., Huang, S., Zhang, B. (2020). Consensus-oriented cloud manufacturing based on blockchain technology: An exploratory study. Pervasive and Mobile Computing, 62, 101113. https://doi.org/10.1016/j.pmcj.2020.101113

50. Yu, C., Zhang, L., Zhao, W., Zhang, S. (2020). A blockchain-based service composition architecture in cloud manufacturing. International Journal of Computer Integrated Manufacturing, 33(7), 701-715. https://doi.org/10.1080/0951192X.2019.1571234

51. Nguyen, N. T., Leu, M. C., Liu, X. F. (2018). RTEthernet: Real-time communication for manufacturing cyberphysical systems. Transactions on Emerging Telecommunications Technologies, 29(7), e3433. https://doi.org/10.1002/ett.3433

52. Cao, Y., Wang, S., Kang, L., Gao, Y. (2016). A TQCS-based service selection and scheduling strategy in cloud manufacturing. The International Journal of Advanced Manufacturing Technology, 82(1-4), 235-251. https://doi.org/10.1007/s00170-015-7350-5

53. Xu, W., Tian, S., Liu, Q., Xie, Y., Zhou, Z., Pham, D. T. (2016). An improved discrete bees algo- rithm for correlation-aware service aggregation optimization in cloud manufacturing. The International Journal of Advanced Manufacturing Technology, 84(1-4), 17-28. https://doi.org/10.1007/s00170-015-7738-2

54. Hu, Y., Zhu, F., Zhang, L., Lui, Y., Wang, Z. (2019). Scheduling of manufacturers based on chaos optimization algorithm in cloud manufacturing. Robotics and Computer-Integrated Manufacturing, 58, 13-20. https://doi.org/10.1016/j.rcim.2019.01.010

55. Luo, Y., Zhang, L., Tao, F., Ren, L., Liu, Y., Zhang, Z. (2013). A modeling and description method of multidimensional information for manufacturing capability in cloud manufacturing system. The International Journal of Advanced Manufacturing Technology, 69(5-8), 961-975. https://doi.org/10.1007/s00170-013-5076-9

56. Yu, Y., Xu, W. (2020). Optimized Configuration of Manufacturing Resources for Middle and Lower Batch Customization Enterprises in Cloud Manufacturing Environment. Complexity, 2020. https://doi.org/10.1155/2020/5619758

57. Chen, T., Wang, Y. C. (2016). Estimating simulation workload in cloud manufacturing using a classifying artificial neural network ensemble approach. Robotics and Computer-Integrated Manufacturing, 38, 4251. https://doi.org/10.1016/j.rcim.2015.09.011

58. Liu, Y. K., Zhang, X. S., Zhang, L., Tao, F. Wang, L. H. (2019). A multi-agent architecture for scheduling in platform-based smart manufacturing systems. Frontiers of Information Technology and Electronic Engineering, 20(11), 1465-1492. https://doi.org/10.1631/FITEE.1900094

59. Zhou, L., Zhang, L., Ren, L., Wang, J. (2019). Real-time scheduling of cloud manufacturing services based on dynamic data-driven simulation. IEEE Transactions on Industrial Informatics, 15(9), 5042-5051. https://doi.org/10.1109/TII.2019.2894111

60. Jiang, H., Liu, C. Y. (2019). Scheduling Optimization of Cloud Resource Supply Chain through Multi-Objective Particle Swarm Optimization. International Journal of Simulation Modelling, 18(1), 163-174. https://doi.org/10.2507/IJSIMM18(1)CO3

61. Talhi, E., Huet, J. C., Fortineau, V., Lamouri, S. (2020). A methodology for cloud manufacturing architecture in the context of Industry 4.0. Bulletin of the Polish Academy of Sciences. Technical Sciences, 68(2). https://doi.org/10.24425/bpasts.2020.131849

62. Tao, F., Cheng, J., Cheng, Y., Gu, S., Zheng, T., Yang, H. (2017). SDMSim: a manufacturing service supply-demand matching simulator under cloud environment. Robotics and computer-integrated manufacturing, 45, 3446. https://doi.org/10.1016/j.rcim.2016.07.001

63. Liang, H., Sun, L. (2019). Improve cloud manufacturing supply chain note-enterprises optimize combination of the Cuckoo search. Concurrency and Computation: Practice and Experience, 31(10), e4764. https://doi.org/10.1002/cpe.4764

64. Lojka, T., Bundzel, M., Zolotová, I. (2016). Service-oriented architecture and cloud manufacturing. Acta polytechnica hungarica, 13(6), 25-44. https://doi.org/10.12700/aph.13.6.2016.6.2

65. Li, C., Zhang, M., He, H., Li, C., Chang, Y. Shang, Y. (2015). Research of Improved ZigBee-based AODVjr Routing Algorithm in Cloud Manufacturing. International Journal of Online Engineering, 11(2). http://dx.doi.org/10.3991/ijoe.v11i2.4252 


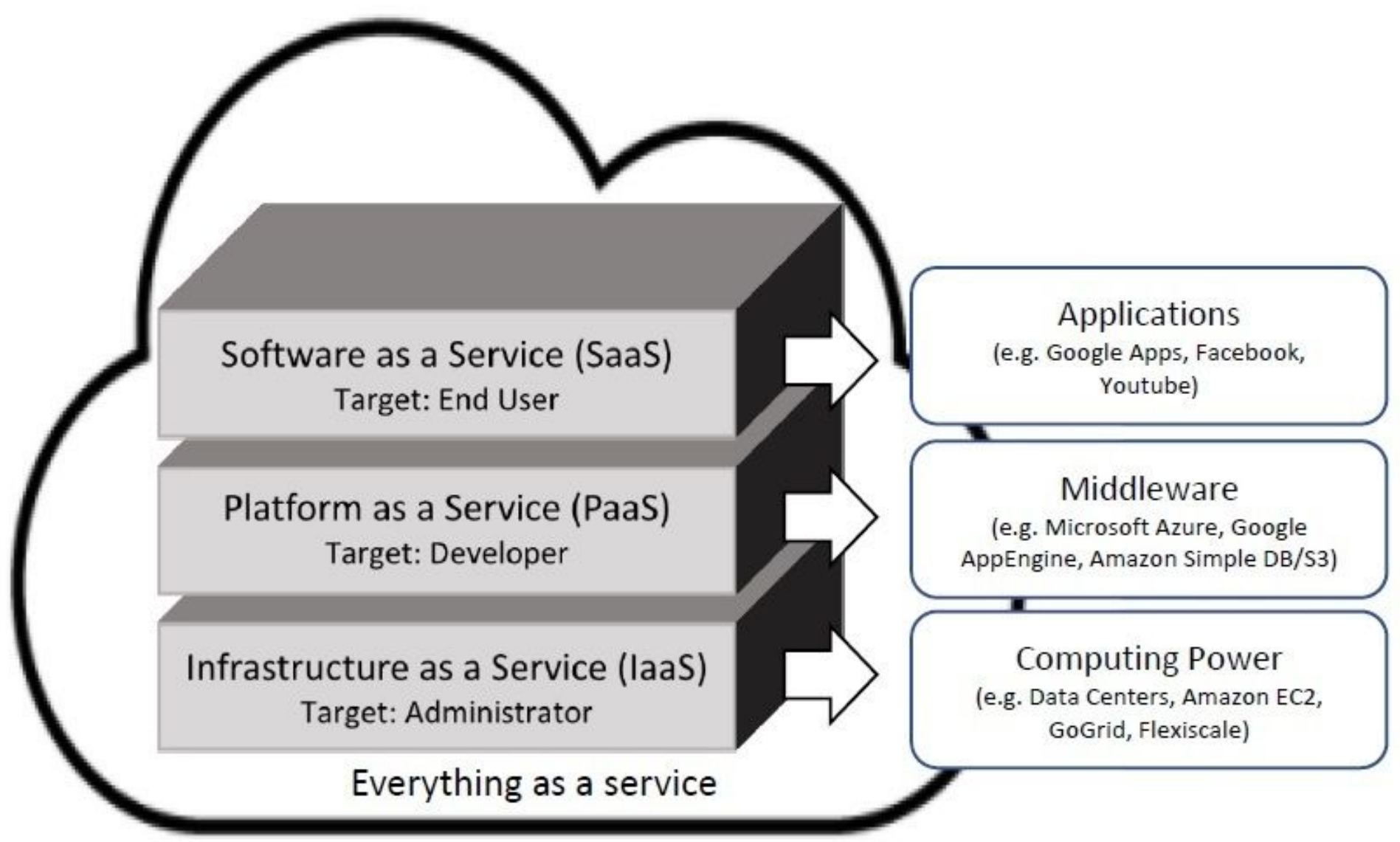

Figure 1

Resources as a service in cloud computing. 


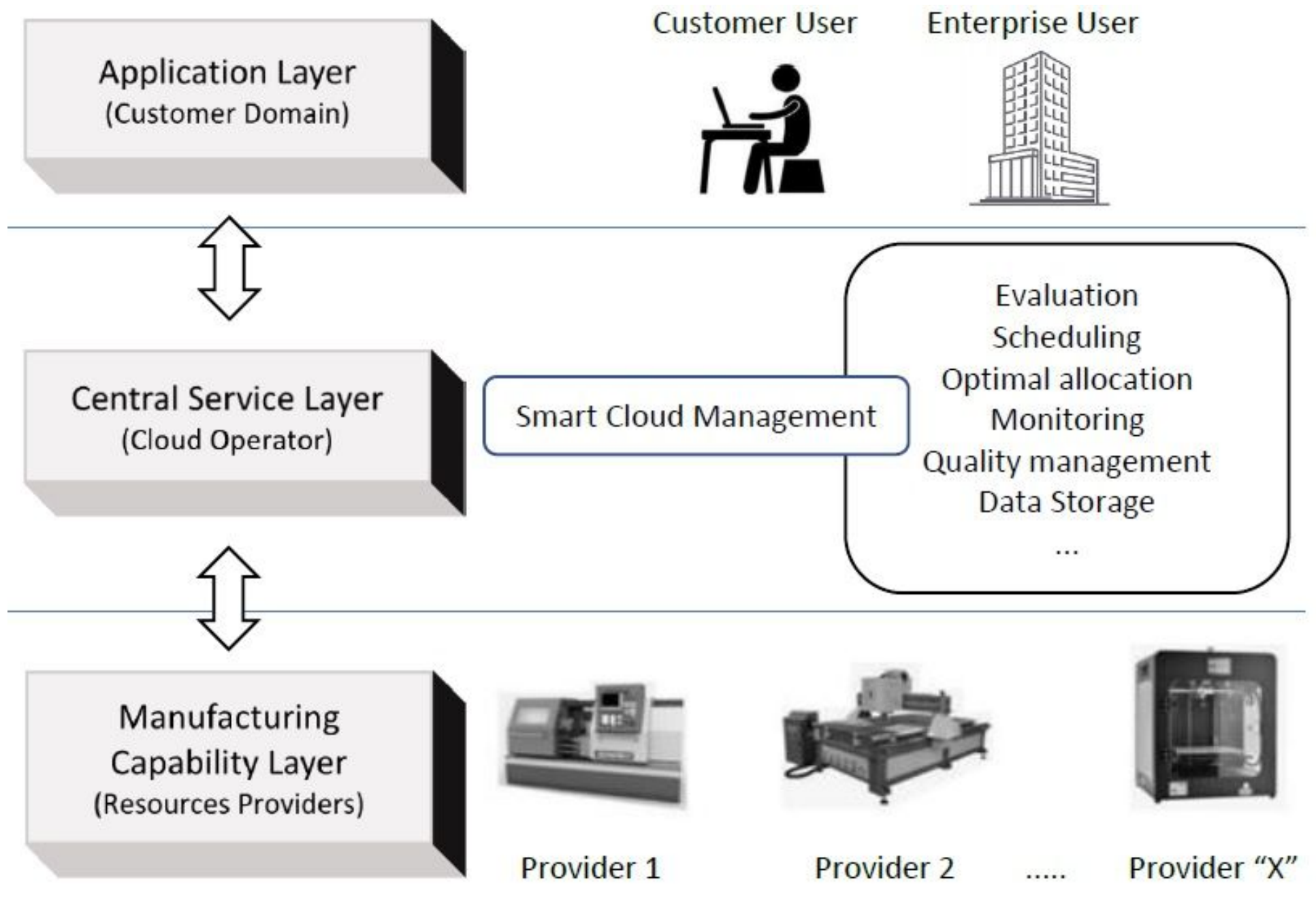

Figure 2

The Cloud Manufacturing architecture model. 


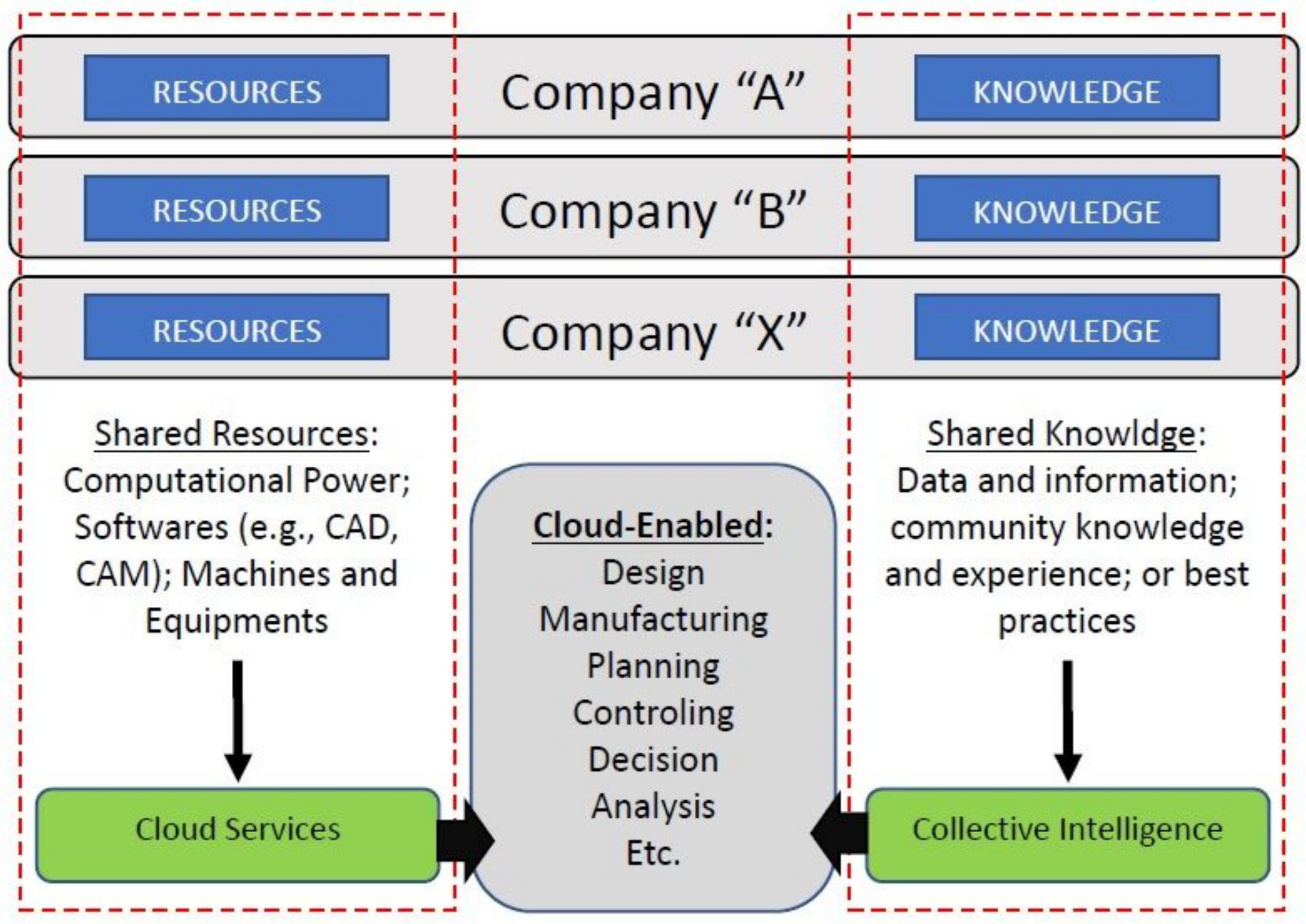

Figure 3

Potentials of resources and knowledge sharing.

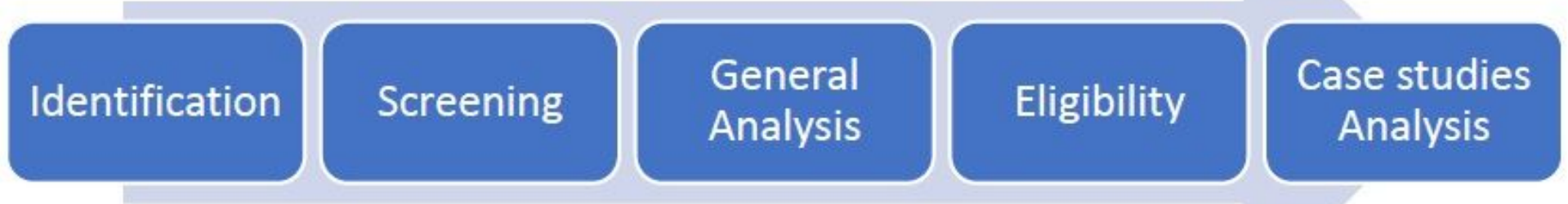

Figure 4

The 5 steps applied in this bibliometric analysis. 


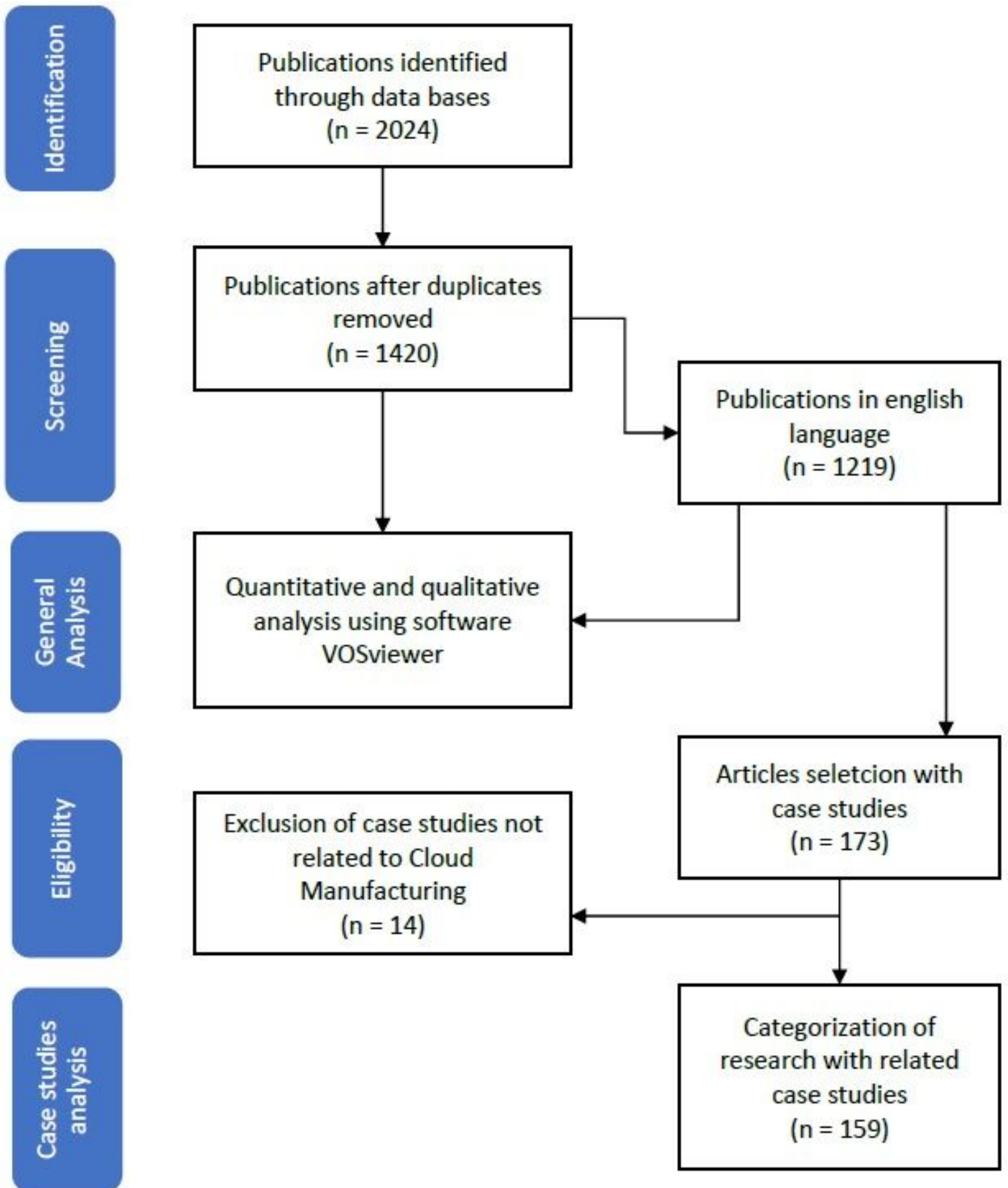

Figure 5

Detailed flowchart of the research methodology. 


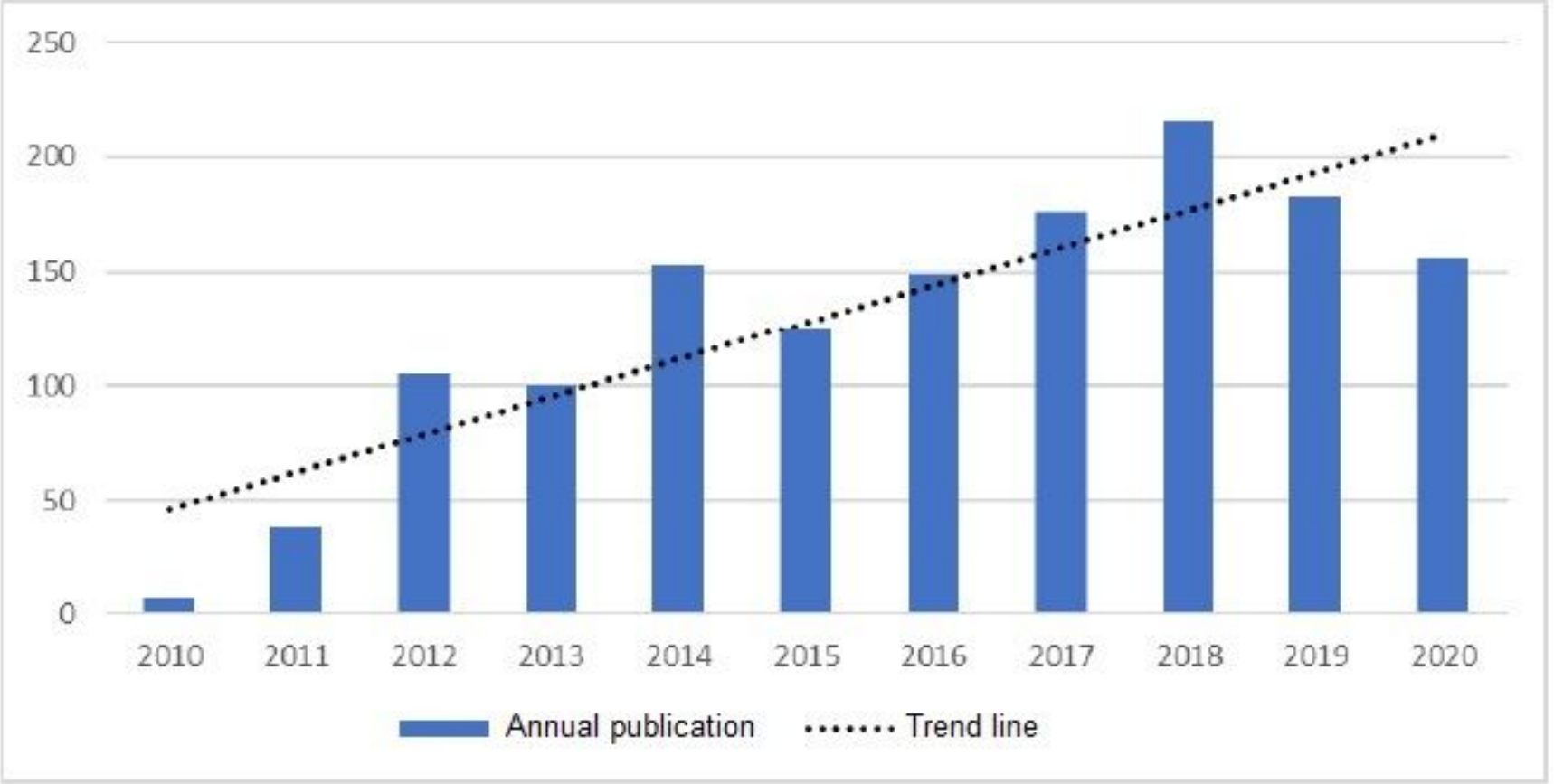

Figure 6

Annual publications and trend about Cloud Manufacturing. 


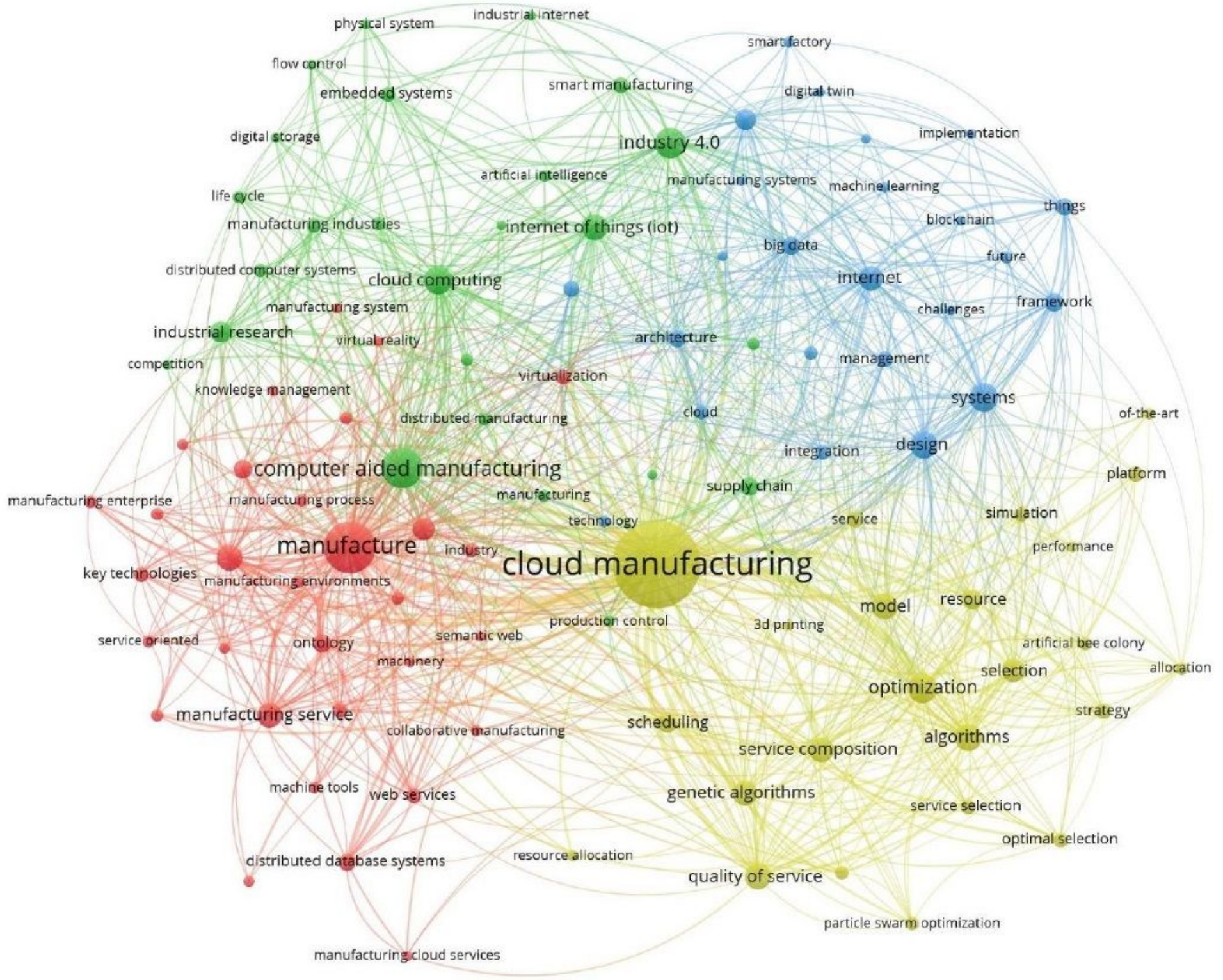

Figure 7

Keywords co-occurrence map. 


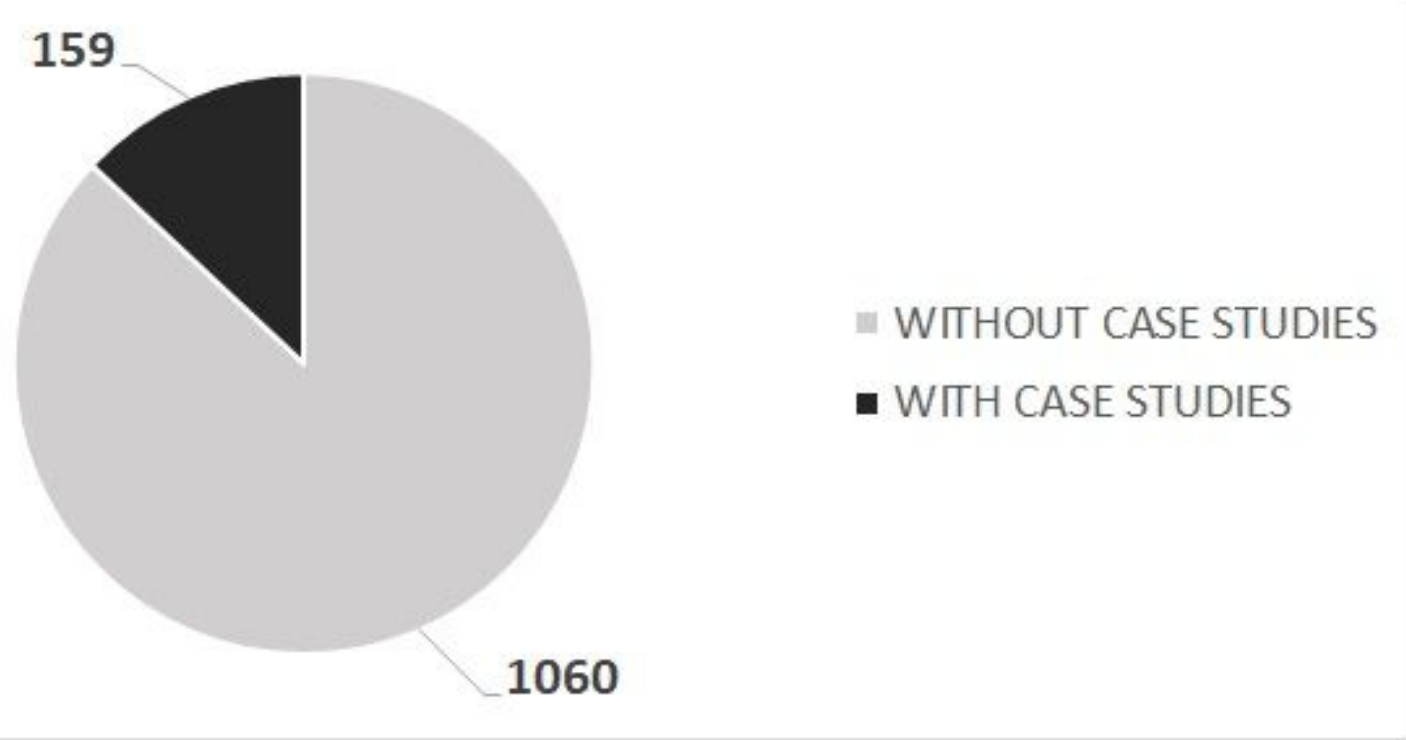

Figure 8

Publications in English language, with and without case studies.

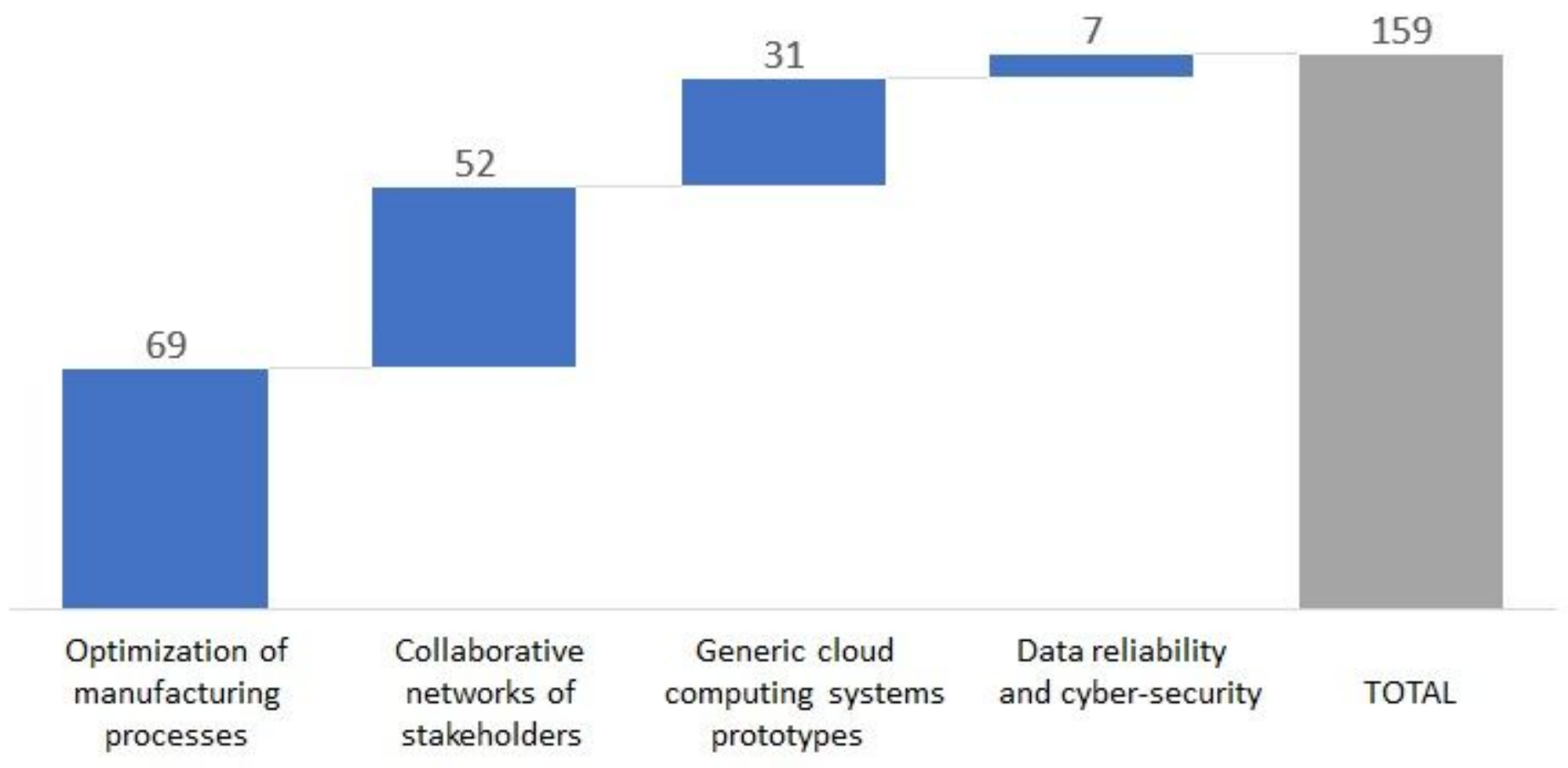

Figure 9

Case studies classified by their approach. 